\title{
Spectral-element simulations of variable-density turbulent flow in a plane channel
}

\author{
Vladimir Ryzhenkov ${ }^{1,2}$, Vladislav Ivashchenko ${ }^{1,2}$, Ricardo Vinuesa ${ }^{3}$ and Rustam \\ Mullyadzhanov ${ }^{1,2}$ \\ ${ }^{1}$ Institute of Thermophysics SB RAS, 630090, Novosibirsk, Russia \\ ${ }^{2}$ Novosibirsk State University, 630090, Novosibirsk, Russia \\ ${ }^{3}$ Linne FLOW Centre, KTH Mechanics and Swedish e-Science Research Centre, SE-100 44, \\ Stockholm, Sweden
}

\begin{abstract}
We perform Large-eddy simulations (LES) of the turbulent flow in a channel with isothermal heated walls with the temperature ratio equal to 2. The variable properties of the fluid are accounted for by using the low Mach number approximation. The Reynolds number based on the bulk velocity, half-width of the channel, density and dynamic viscosity near the cold wall is 6800 . We study the effect of spatial resolution on the accuracy of Large-eddy simulations with dynamic Smagorinsky model. The very good agreement of LES results is shown even for the coarse meshes which is attributed to the high accuracy of the spectral method.
\end{abstract}

\section{Introduction}

The accuracy of numerical simulations hardly relies on the chosen discretization method. In the present work we employ spectral-element approach [1] which combines the accuracy of spectral methods and flexibility of finite-element approach allowing unstructured grids. We further assess the performance of the nek5000 code in the low Mach approximation targeting the turbulent channel flow $[2,3]$.

\section{The governing equations and computational details}

The fluid motion at low speed can be described within the low Mach number approximation with the non-dimensional mass, momentum and energy transport equations $[2,4]$ :

$$
\frac{\partial \rho}{\partial t}+\frac{\partial}{\partial x_{j}}\left(\rho u_{j}\right)=0
$$




$$
\begin{gathered}
\frac{\partial\left(\rho u_{i}\right)}{\partial t}+\frac{\partial}{\partial x_{j}}\left(\rho u_{j} u_{i}\right)=-\frac{\partial p}{\partial x_{j}}+\frac{1}{R e} \frac{\partial \sigma_{i j}}{\partial x_{j}} \\
C_{p}\left(\frac{\partial(\rho T)}{\partial t}+\frac{\partial\left(\rho u_{j} T\right)}{\partial x_{j}}\right)=\frac{1}{\operatorname{Re} \cdot \operatorname{Pr}} \frac{\partial}{\partial x_{j}}\left(k \frac{\partial T}{\partial x_{j}}\right)+\frac{\gamma-1}{\gamma} \frac{d p_{0}}{d t},
\end{gathered}
$$

where $\rho, T, \boldsymbol{u}, p$ are the non-dimensional density, temperature, velocity and hydrodynamic pressure fields respectively, $\sigma_{i j}=\mu\left(\partial u_{i} / \partial x_{j}+\partial u_{j} / \partial x_{i}-(2 / 3) \delta_{i j} \partial u_{k} / \partial x_{k}\right)$ is the viscous stress tensor, $\gamma$ is the ratio of specific heats and $p_{0}$ is the thermodynamic pressure, which does not depend on space and is determined from a proper equation of state. All the variables are normalized using the reference state $u^{\text {ref }}, T^{\text {ref }}=p_{0}^{\text {ref }} / \rho^{\text {ref }}, C_{p}^{\text {ref }}=$ $C_{p}^{*}\left(T^{r e f}\right), \mu^{r e f}=\mu^{*}\left(T^{r e f}\right)$ and $k^{r e f}=k^{*}\left(T^{r e f}\right)$, where the superscript $*$ represent dimensional quantities [2]. The Reynolds and Prandtl numbers are defined as follows $R e=u^{r e f} L^{r e f} / v^{r e f}, P r=\rho^{r e f} C_{p}^{r e f} v^{r e f} / k^{r e f}$. Note that $C_{p}=1$ in Eq. (3). We consider the ideal gas equation of state:

$$
p_{0}=\rho T
$$

The volume integration of (4) gives:

$$
p_{0}=\frac{M}{\int_{V}(1 / T) d V},
$$

where $M=\int_{V} \rho d V$ is the total mass of fluid in the computational domain.

We perform Large-eddy simulations (LES) of the Navier-Stokes equations where the spatial low-pass filtering denoted by an overline is applied to the equations of motion eq. (1)-(3):

$$
\bar{u}(\boldsymbol{x}, t)=\int G(\boldsymbol{x}-\boldsymbol{r}) \boldsymbol{u}(\boldsymbol{r}, t) d \boldsymbol{r},
$$

where $G$ is the filter function. It is common to use the Favre-averaging in order to preserve the form of equations:

$$
\widetilde{f}=\overline{\rho f} / \bar{\rho}
$$

Filtered equations contain unclosed terms $-\partial \tau_{k l} / \partial x_{k}$ and $-\partial q_{k} / \partial x_{k}$ in the right-hand side of momentum and energy equations, respectively, corresponding to the sub-grid scale stresses and heat flux. These terms have the following form:

$$
\tau_{k l}=\bar{\rho}\left(\widetilde{u_{k} u_{l}}-\widetilde{u_{k}} \widetilde{u_{l}}\right), \quad q_{k}=\bar{\rho}\left(\widetilde{u_{k} T}-\widetilde{u_{k}} \tilde{T}\right)
$$

The Boussinesq hypothesis and Smagorinsky model are used to express sub-grid terms:

$$
\tau_{k l}-\tau_{m m} \delta_{k l} / 3=-2 \bar{\rho} v_{t}\left(\tilde{S}_{k l}-\tilde{S}_{m m} \delta_{k l} / 3\right)=-2 C \bar{\rho} \Delta^{2}|\tilde{S}|\left(\tilde{S}_{k l}-\tilde{S}_{m m} \delta_{k l} / 3\right),
$$

where $\tilde{S}_{k l}=\left(\partial \tilde{u}_{k} / \partial x_{l}+\partial \tilde{u}_{l} / \partial x_{k}\right) / 2,|\tilde{S}|=\sqrt{2 \tilde{S}_{k l} \tilde{S}_{k l}}, v_{t}=C \Delta^{2}|\tilde{S}|$ is the eddy viscosity, $\Delta$ the local filter size. The term $\tau_{m m}$ is lumped into the pressure field. The Smagorinsky constant $\mathrm{C}$ is defined according to the dynamic procedure [5]: 


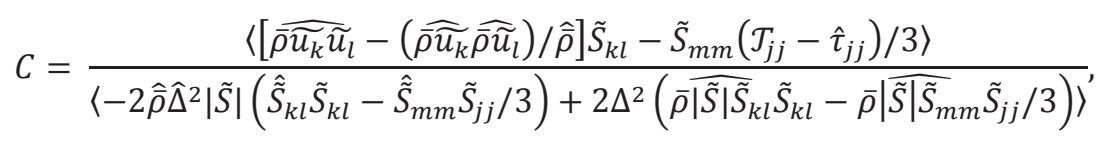

where the brackets $\langle\ldots\rangle$ denote the averaging along homogeneous directions. Dynamic procedure implies the introduction of the test filter $\widehat{\Delta}=2 \Delta$. The test-filtered stresses used in Eq. (11) are defined as:

$$
\mathcal{T}_{k l}=\widehat{\rho u_{k} u_{l}}-\left(\widehat{\rho u_{k}} \widehat{\rho u_{l}} / \overline{\bar{\rho}}\right)
$$

Similarly, the heat flux vector is modeled as follows:

$$
q_{k}=-\frac{\bar{\rho} v_{t}}{P r_{t}} \frac{\partial \tilde{T}}{\partial x_{k}}
$$

The turbulent Prandtl number is defined in a similar manner:

$$
\operatorname{Pr}_{t}=C\left\langle\widehat{\Delta}^{2} \hat{\bar{\rho}}|\hat{\tilde{S}}| \frac{\partial \hat{\tilde{T}}}{\partial x_{k}} \frac{\partial \tilde{T}}{\partial x_{k}}-\left(\Delta^{2} \bar{\rho}|\widehat{S}| \frac{\partial \tilde{T}}{\partial x_{k}}\right) \frac{\partial \tilde{T}}{\partial x_{k}}\right\rangle \times\left\langle\left(\frac{\widehat{\bar{\rho} \widetilde{u}_{k}} \widehat{\bar{\rho} \tilde{T}}}{\hat{\bar{\rho}}}-\widehat{\bar{\rho} \widetilde{u_{k}} \tilde{T}}\right) \frac{\partial \tilde{T}}{\partial x_{k}}\right\rangle^{-1}
$$

The above described governing equations are implemented in the nek5000 code [6] with a spatial discretization based on the spectral-element method using Lagrange polynomials. The Navier-Stokes equations are cast in a weak form and discretized in space by means of Galerkin approximation using $\boldsymbol{N}^{\text {th }}$-order Lagrange polynomial interpolants on the GaussLobatto-Legendre points for the velocity field and pressure $\left(\mathbb{P}_{N}-\mathbb{P}_{N}\right.$ formulation). In the present work the polynomial order $\mathrm{N}$ is set to 7 . The semi-implicit time-stepping scheme is third-order accurate.

\section{Problem formulation and results of simulations}

We study a turbulent flow between two infinite parallel isothermal heated walls at a Reynolds number of $R e_{c}=\rho_{c} U_{b} H / \mu_{c}$, where $U_{b}$ is the bulk velocity, $H$ is the half-width of the channel, $\rho_{c}, \mu_{c}$ the density and dynamic viscosity near the cold wall providing the reference values for the non-dimensionalization of Navier-Stokes equations described above. A rectangular computational domain of the size $L_{x} \times L_{y} \times L_{z}=2 \pi H \times 2 H \times \pi H$ is used where $x \in[0,2 \pi H]$ is the streamwise, $y \in[-H, H]$ the wall-normal and $z \in$ $[0, \pi H]$ the spanwise coordinates. The planes $y= \pm H$ correspond to the position of parallel walls. The infinite flow domain is modelled by periodic boundary conditions in $x$ and $z$ directions. No-slip condition and constant temperature for hot upper $\left(T=T_{\mathrm{h}}\right)$ and cold lower $\left(T=T_{\mathrm{c}}\right)$ wall are imposed. A dynamically adjusted body force is added in equations (1) and (3) to keep a constant volume flux through the plane $y-z$. The dynamic viscosity and thermal conductivity depend on the temperature according to the Sutherland's law:

$$
\mu(T)=T^{3 / 2}\left[1+S_{\mu}\right] /\left[T+S_{\mu}\right], \quad k(T)=T^{3 / 2}\left[1+S_{k}\right] /\left[T+S_{k}\right],
$$

where $S_{\mu}=0.368, S_{k}=0.648$. We consider a mild temperature difference, i.e. $T_{\mathrm{h}} / T_{\mathrm{c}}=2$. Note that using these relations the Reynolds number calculated with hot wall characteristics correspond to $R e_{h}=\rho_{h} U_{b} H / \mu_{h}=2081$. The difference between instantaneous temperature fields for constant- [7] and variable-density flows is shown in Fig. 1. 
We consider different meshes for variable-density LES simulations with $N_{S E}=20 \times$ $16 \times 16$ (fine) and $14 \times 10 \times 10$ (coarse), where $N_{S E}$ is the number of spectral elements along each direction $(x, y, z)$, corresponding to $N_{\text {total }}=2.6 \times 10^{6}$ and $0.72 \times 10^{6}$, with $N_{\text {total }}$ being total number of points. Both meshes satisfy the near-wall LES criteria.

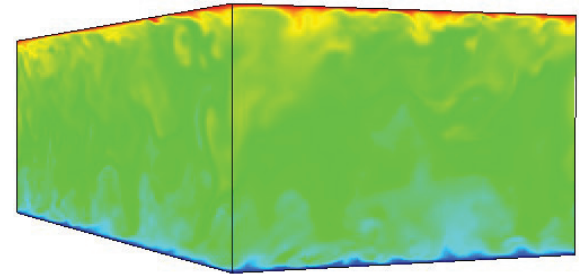

a

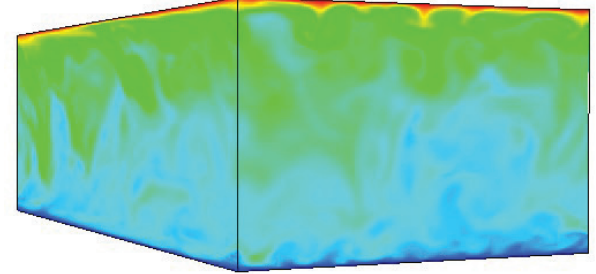

$\mathrm{b}$

Fig. 1. Instantaneous temperature field for (a) the constant-density flow, (b) variable-density flow.

Previously we have compared constant-density simulations with the data from the literature finding excellent agreement for velocity and temperature fields. Figure 2 shows the comparison of $\boldsymbol{u}, T$ and their fluctuations for coarse and fine LES of variable-density flow. Note the inevitable asymmetry in profiles with respect to $y \rightarrow-y$ due to the effect of density/temperature on the velocity field.
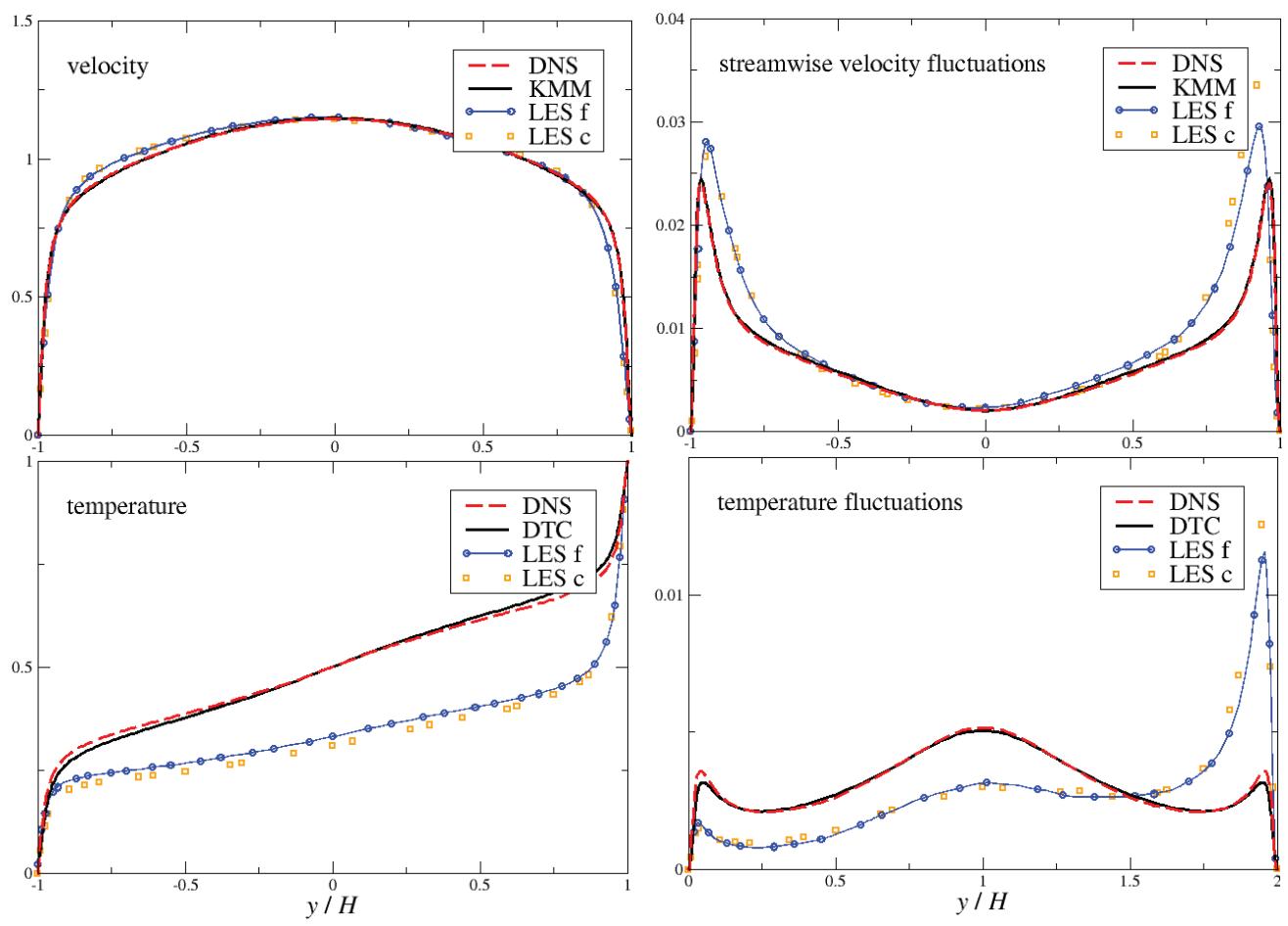

Fig. 2. Black and red dashed line shows the constant-density results [7-9]. Symbols "f" and "c" denote the coarse and fine LES profiles for the variable-density flow respectively.

\section{Conclusions}


We have performed a series of Large-eddy simulations of a variable-density turbulent channel flow at the Reynolds number of 6800 using the spectral-element method. The difference between constant- and variable density flow has been outlined. The very good agreement of LES results is shown even for the coarse mesh which is attributed to the high accuracy of the spectral method.

This work is funded by RFBR grants No. 15-08-06166 and No. 14-08-01233a. The computational resources are provided by Siberian Supercomputer Center SB RAS (Novosibirsk) and Supercomputing Center of the Novosibirsk State University (Novosibirsk).

\section{References}

1. M. O. Deville, P. F. Fischer, E. H. Mund, High-order Methods for Incompressible Fluid Flow (Cambridge University Press, New York, 2004)

2. F. C. Nicoud, Numerical study of a channel flow with variable properties (CTR, Annu. Res. Briefs, 1998)

3. A. Tyliszczak, Comp. Fluids 127, 131-145, 2016

4. S. Paolucci, On the filtering of sound from the Navier-Stokes equations (SAND828253, Sandia National Laboratories, Livermore, 1982)

5. P. Moin, K. Squires, W. Cabot, S. Lee, Phys. Fluids A 3, 2746, 1991

6. P. Fischer, J. Lottes, S. Kerkemeier, Nek5000 web page (http://nek5000.mcs.anl.gov)

7. V. Ryzhenkov, V. Ivashchenko, R. Vinuesa, R. Mullyadzhanov, J. Phys.: Conf. Ser. 754 (6), 062009, 2016

8. R. D. Moser, J. Kim, N. N. Mansour, Phys. Fluids 11 (4), 943-945, 1999

9. S. Dharmarathne, M. Tutkun, G. Araya, L. Castillo, Eur. J. Mech.-B/Fluids 55, 259-271, 2016 